\title{
The value of D-dimer, ESR and CRP in the Diagnosis of Deep Vein Thrombosis of Lower Limbs After Hip and Knee Joint Replacement
}

\section{Xinchao Fan}

HEBEINU: Hebei North University https://orcid.org/0000-0002-8795-1444

\section{Kai Zhang}

Hospital Provincial

\section{Xilong Sun}

Hospital Provincial

\section{Xiangrui Duan}

North China University of Science and Technology

\section{Dadi Wan}

North China University of Science and Technology

\section{Ye Yuan}

HEBEINU: Hebei North University

\section{Yibin Zhou}

Hospital Provincial

Changmao Qiu

Hospital Provincial

Wenyi Li

Hebei Provincial People's Hospital

Xicheng Li ( $\sim$ lixicheng9999@163.com )

Hebei Provincial People's Hospital

\section{Research article}

Keywords: Joint replacement, DVT, D-dimer, ESR, CRP

Posted Date: February 4th, 2021

DOI: https://doi.org/10.21203/rs.3.rs-171633/v1

License: (c) (1) This work is licensed under a Creative Commons Attribution 4.0 International License.

Read Full License 


\section{Abstract}

Objective: To investigate the diagnostic value of D-dimer, ESR and CRP in deep vein thrombosis (DVT) of lower extremity after hip and knee replacement.

Methods: Clinical data of 216 patients who underwent hip and knee replacement in our hospital from January 2018 to November 2020 were retrospectively analyzed. Among them, there were 86 patients with lower extremity deep vein thrombosis and 130 patients without lower extremity deep vein thrombosis. Preoperative blood D-dimer, prothrombin time, fibrinogen content, erythrocyte sedimentation rate (ESR), Creactive protein (CRP), albumin and hemoglobin levels were collected. Chi-square test was used to compare the rate of deep vein thrombosis in lower limbs. The PSM propensity score matching method was used to select 86 patients from 130 patients without lower extremity deep vein thrombosis (DVT) as control group. The levels of D-dimer, albumin and hemoglobin in 172 patients were compared, and the diagnostic efficacy of D-dimer, ESR and CRP in the diagnosis of lower extremity deep vein thrombosis after hip and knee replacement was analyzed by ROC curve.

Results: ROC curve analysis results showed that: The optimal cut-off values of D-dimer, prothrombin time, ESR, CRP and age for the diagnosis of DVT of lower extremity after hip replacement were $1.745 \mathrm{mg} / \mathrm{L}$, $10.850 \mathrm{~s}, 15.500 \mathrm{~mm} / \mathrm{h}, 2.375 \mathrm{mg} / \mathrm{L}$ and 72 years old, respectively. The sensitivity was $71.8 \%, 66.7 \%$, $61.5 \%, 94.9 \%, 71.8 \%$, the specificity was $74.1 \%, 66.7 \%, 70.4 \%, 33.3 \%, 100 \%$, and the area under the curve was $0.746,0.683,0.658,0.651,0.869$. The optimal truncation value of D-dimer for the diagnosis of DVT of lower extremity after knee replacement was $0.285 \mathrm{mg} / \mathrm{L}$, the sensitivity was $78.7 \%$, the specificity was $44.1 \%$, and the area under the curve was 0.622 .

Conclusion: The diagnostic value of D-dimer in the formation of lower extremity DVT after knee arthroplasty is higher than that after hip arthroplasty, but the diagnostic value of prothrombin time, ESR, CRP and age in the formation of lower extremity DVT after hip arthroplasty is higher than that after knee arthroplasty.

\section{Background}

Artificial joint replacement is an important method for the treatment of late hip and knee joint pain and dysfunction. Joint replacement can quickly and effectively reconstruct joint function, eliminate joint pain, correct deformity and dysfunction [[1]]. With the development of the aging population, the number of patients with joint degenerative diseases has increased, and artificial joint replacements have become more common. Many county hospitals have carried out joint replacements. At the same time, postoperative complications of joint replacement have also increased. Deep Vein Thrombosis (DVT) formation is a common perioperative complication of orthopedic hospitalized patients, and its incidence can reach $10 \%-80 \%$ [[2]]. The main causes of DVT in the lower limbs are the perioperative blood in patients with hypercoagulable state and slow braking blood flow in the lower limbs. The formation of DVT in the lower limbs can increase the patient's pain, lengthen the hospital stay, increase medical 
expenses, and cause pulmonary embolism once it falls off. In severe cases, it can lead to death [[3]]. Perfect preoperative examination, correct application of anticoagulant drugs, and improve blood circulation can effectively prevent deep vein thrombosis of lower limbs, thereby reducing the burden on patients. Preoperative blood test is a routine test for artificial joint replacement. The blood D-dimer, fibrinogen and other levels can effectively assess the probability of deep vein thrombosis in the lower limbs after surgery. The author analyzed the clinical data of 216 patients who underwent hip and knee arthroplasty in our hospital from January 2018 to November 2020, and explored the diagnostic value of D-dimer in lower limb DVT after hip and knee arthroplasty.

\section{Materials And Methods}

1. normal information

1. Inclusion criteria: $\triangle \mathrm{Patients}$ underwent hip and knee joint replacement surgery in our hospital from January 2018 to November 2020; 『First hip and knee joint replacement surgery; 『No lower extremity thrombosis before surgery; $\mathbb{}$ Complete medical records, And approved by the patient and the hospital ethics committee.

2. Exclusion criteria: $\triangle$ Patients with a history of deep vein thrombosis or varicose veins in the lower

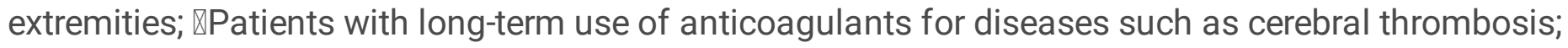
$\triangle$ Patients with a history of malignant tumors or tuberculosis; $₫$ Patients with rheumatic diseases or blood

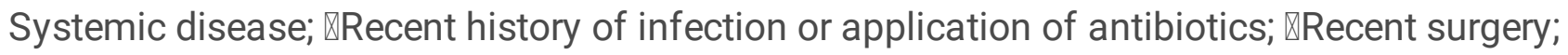

3. According to the inclusion and exclusion criteria, a total of 216 patients were included. There were 69 males and 147 females, aged 46-94 years old, with an average of $(70.06 \pm 10.03)$ years old. 77 cases of total knee replacement, 31 cases of unicondyle replacement, 35 cases of total hip replacement, and 73 cases of hemi-hip replacement. Among them, 86 patients had deep venous thrombosis of lower extremities after operation, and 130 patients had no deep venous thrombosis of lower extremities after operation. The diagnosis of DVT in the lower limbs is based on the postoperative deep vein color Doppler ultrasound.

\section{Detection of research indicators}

All study patients collected and analyzed D-dimer, prothrombin time, fibrinogen content, Erythrocyte Sedimentation Rate (ESR), C-Reactive Protein (CRP), white Protein and hemoglobin levels. Normal value range: D-dimer: 0-0.55mg/L, prothrombin time: $9.8-12.1$ seconds, fibrinogen content: $2-4 \mathrm{~g} / \mathrm{L}, \mathrm{ESR}: \mathrm{mm} / \mathrm{h}$, CRP: 0-10mg/ L, albumin: 40-55g/L, hemoglobin: $130-175 \mathrm{~g} / \mathrm{L}$.

Third, statistical methods

Use SPSS23.0 statistical software for statistical analysis. Measurement data conforming to the normal distribution are expressed by $\pm \mathrm{s}$, and comparison between groups is performed by $t$ test; measurement data not conforming to the normal distribution is expressed by median \pm quartile, and comparison 
between the two groups is performed by Mann-Whitney $U$ test; The data is expressed in frequency, and the $x$ [2] test is used for comparison between groups. $P<0.05$ indicates that the difference is statistically significant.

\section{Result}

1. Comparison of the incidence of lower limb venous thrombosis after different replacement types

Among the 216 patients, 35 had total hip arthroplasty, 8 had deep venous thrombosis after operation, the incidence of thrombosis was 22.9\%; 74 had hemi-hip arthroplasty, and 34 had deep venous thrombosis after the operation, and the incidence of thrombosis $46.6 \% ; 77$ cases of total knee arthroplasty, 25 cases of postoperative deep venous thrombosis of the lower extremities, the incidence of thrombosis was $32.5 \%$; 31 cases of unicondyle replacement, 19 cases of postoperative deep venous thrombosis of the lower extremities, and the incidence of thrombosis $61.3 \%$; See Table 1.

2. Based on age, gender and replacement type as the standard, using the PSM propensity score matching method, 86 patients were selected as the control group from 130 patients without deep vein thrombosis after operation, and the general information of 172 patients; see Table 2.

3. Comparison of the levels of D-dimer, albumin and hemoglobin between the thrombus group and the control group

The D-dimer level of patients in the thrombus group was $0.98(0.378 \sim 3.463) \mathrm{mg} / \mathrm{L}$, which was higher than the control group by $0.43(0.238 \sim 0.893) \mathrm{mg} / \mathrm{L}, \mathrm{P}=0.000$, the difference was statistically significant; the albumin of the patients in the thrombosis group was $(38.806 \pm 4.625) \mathrm{g} / \mathrm{L}$, lower than the control group $(41.003 \pm 3.824) \mathrm{g} / \mathrm{L}, \mathrm{P}=0.001$, the difference was statistically significant; the hemoglobin of the thrombosis group was $(128.663 \pm 15.776) \mathrm{g} / \mathrm{L}$, lower than the control group $(134.430 \pm 12.842) \mathrm{g} / \mathrm{L}, \mathrm{P}=$ 0.009 , the difference is statistically significant; see Table 3.

Comparison of D-mer, albumin and hemoglobin levels in hip and knee replacement patients in thrombosis group

The D-dimer level of hip replacement patients was $3.25(1.240 \sim 8.350) \mathrm{mg} / \mathrm{L}$, which was higher than the D-dimer level of knee replacement patients by $0.45(0.290 \sim 0.970) \mathrm{mg} / \mathrm{L}, \mathrm{P}=0.000$, the difference was statistically significant Scientific significance; the albumin level of hip replacement patients was (36.199 $\pm 4.569) \mathrm{g} / \mathrm{L}$, which was lower than that of knee replacement patients $(40.969 \pm 3.4269) \mathrm{g} / \mathrm{L}, \mathrm{P}=0.000$, the difference was statistically significant; hip joint The hemoglobin level of replacement patients was $(125.897 \pm 16.665) \mathrm{g} / \mathrm{L}$, which was lower than that of knee replacement patients $(130.957 \pm 14.785) \mathrm{g} / \mathrm{L}$, $P=0.140$, the difference was not statistically significant; see Table 4.

The diagnostic efficacy of D-dimer, prothrombin time, ESR, CRP, and age on the formation of DVT in lower limbs after hip replacement 
ROC curve analysis results show that the best cut-off value of D-dimer in the diagnosis of lower extremity DVT after hip replacement is $1.745 \mathrm{mg} / \mathrm{L}$, sensitivity is $71.8 \%$, specificity is $74.1 \%$, and area under the curve is 0.746 (95\%). Cl: $0.622 \sim 0.870), \mathrm{P}=0.001$, which is statistically significant; the best cutoff value of prothrombin time is 10.850 s, the sensitivity is $66.7 \%$, the specificity is $66.7 \%$, and the area under the curve is 0.683 ( $95 \% \mathrm{Cl}: 0.551 \sim 0.816$ ), $\mathrm{P}=0.012$, which is statistically significant; the best ESR cutoff value is $15.500 \mathrm{~mm} / \mathrm{h}$, the sensitivity is $61.5 \%$, the specificity is $70.4 \%$, and the area under the curve is 0.658 (95\%Cl: $0.526 \sim 0.790), \mathrm{P}=0.030$, statistically significant; the best cut-off value of CRP is 2.375 $\mathrm{mg} / \mathrm{L}$, sensitivity is $94.9 \%$, specificity is $33.3 \%$, area under the curve is 0.651 ( $95 \% \mathrm{Cl}: 0.514 \sim 0.787), \mathrm{P}=$ 0.039 , It is statistically significant; the best cut-off value for age is 72 , the sensitivity is $71.8 \%$, the specificity is $100 \%$, the area under the curve is 0.869 (95\% Cl: $0.778 \sim 0.960), \mathrm{P}=0.000$, which is statistically significant; see Fig. 1.

The diagnostic efficacy of D-dimer, prothrombin time, ESR, CRP, and age on the formation of lower limb DVT after knee arthroplasty

ROC curve analysis results show that the best cut-off value of D-dimer in the diagnosis of lower limb DVT after knee arthroplasty is $0.850 \mathrm{mg} / \mathrm{L}$, sensitivity is $78.7 \%$, specificity is $44.1 \%$, and area under the curve is 0.622 (95\% Cl: $0.515 \sim 0.729), \mathrm{P}=0.032$, statistically significant; the best cut-off value for age is 70.5 years, sensitivity is $34.0 \%$, specificity is $86.4 \%$, area under the curve is 0.589 (95\%Cl: $0.476 \sim 0.703), \mathrm{P}=$ 0.115 , no statistical significance; see Fig. 2.

\section{Discuss}

D-dimer is a specific degradation product of fibrinogen that is activated to form cross-linked fibrin and then hydrolyzed by plasmin [[4]]. It is the simplest fibrin degradation product and its main source The cross-linked fibrin clot dissolved by plasmin [[5]], its concentration can change with factors such as trauma, surgery, pregnancy, thrombosis, etc., to reflect the body's anticoagulation system and fibrinolytic system. Zhang Chengqing and other studies have shown that D-dimer can be used for the diagnosis of deep vein thrombosis and pulmonary embolism in the lower extremities [[6]], Jiang Y[[7]] reported the abnormal increase of D-dimer and the probability of thrombosis And the size is positively correlated, but some scholars have also shown that D-dimer has poor specificity in predicting thrombosis in the body [[8]]. This study showed that D-dimer [3.25 $(1.240 \sim 8.350) \mathrm{mg} / \mathrm{L}]$ in thrombus group hip joint replacement patients was higher than that of knee replacement patients [0.45 $(0.290 \sim 0.970) \mathrm{mg} / \mathrm{L}]$, and D-dimer The best cut-off value $(1.745 \mathrm{mg} / \mathrm{L})$ for diagnosing the formation of lower limb DVT after hip replacement is higher than the best cut-off value $(0.850 \mathrm{mg} / \mathrm{L})$ for knee replacement. This may be because most hip replacements are femoral neck fractures and trauma, Stress causes higher D-dimer. This study showed that the sensitivity (78.7\%) of D-dimer in diagnosing lower limb DVT after knee arthroplasty is higher than that of hip joint $(71.8 \%)$ and the specificity $(44.1 \%)$ is less than hip joint $(74.1 \%)$. The low specificity of Ddimer in diagnosing lower extremity DVT after knee arthroplasty may be due to: $\triangle \mathrm{D}$-dimer level changes are not limited to lower extremity venous thrombotic diseases, but also manifested in coronary heart 
disease, cerebral infarction and other diseases[ [9]]; 『Patients were bedridden for a long time after replacement; $\otimes T$ The number of patients enrolled was limited.

Erythrocyte Sedimentation Rate (ESR) refers to the sinking speed of red blood cells in the serum in a static state, which is mainly affected by the negative charge on the cell membrane surface and decreases in a dispersed manner. When the negative charge on the cell membrane surface is affected, the erythrocyte sedimentation rate becomes abnormal, and its speed Accelerated, the red blood cell sedimentation rate increases [[10]], and ESR is mostly used as an indicator of inflammation and tumor. Creactive protein (CRP) is an acute reactive protein synthesized by the liver. Under normal circumstances, the body's CRP content is very small. When the body has inflammation or trauma, the CRP level will increase sharply. It is usually used as the main indicator to judge inflammation and infection. [[11]]. This study showed that the best cut-off values of ESR and CRP for diagnosing lower limb DVT after hip replacement were $15.500 \mathrm{~mm} / \mathrm{h}$ and $2.375 \mathrm{mg} / \mathrm{L}$, respectively, the sensitivity was $61.5 \%$ and $94.9 \%$, and the specificity was $70.4 \%$ and $33.3 \%$, respectively. The areas under the curve are 0.658 and 0.651 , respectively, which indicates that ESR and CRP can be used as diagnostic indicators for the formation of lower limb DVT after hip replacement. Chen Xiaolan [[12]] and other authors also found that ESR is significantly associated with deep vein thrombosis of the lower extremities. Foreign scholars believe that CRP can activate the body's complement system and cause vascular endothelial damage, induce the body to form a hypercoagulable state, and increase the risk of DVT [[13]]. The results of these studies are consistent with this study. This may be because: 『lnflammation is closely related to coagulation function, infection can promote the damage of the body's vascular endothelial cells, and then make the coagulation-fibrinolysis system more disordered [[14]]; \The body's inflammatory state can promote the crystallization of urate in joints Intraluminal deposition, which in turn promotes deep vein thrombosis [[15]]. This study showed that the best cut-off value for DVT formation of lower limbs after hip arthroplasty was 72 years old, the sensitivity was $71.8 \%$, the specificity was $100 \%$, and the area under the curve was 0.869 . Studies have reported that the risk of DVT in elderly patients is 6 times that of nonelderly patients [[16]]; Ahl et al. [[17]] believe that after the age of 40, every 10 years of age, the incidence of DVT will increase twice, when the age is over 80 years old Later, the incidence increased more significantly. This may be because elderly patients have less exercise, relatively slow blood flow, and high coagulation factors, which promote a high risk of thrombosis.

The shortcomings of this study: $\otimes$ The prothrombin time, ESR, CRP, and age are far behind the hip joint in the diagnosis of lower limb DVT after knee arthroplasty; \the number of cases is limited, which may affect the reliability of the results; $\nabla$ this The research adopts retrospective research, and the inherent defects of retrospective research may affect the reliability of the results to a certain extent.

In summary:D-dimer has differences in the diagnosis of DVT formation after hip and knee arthroplasty. Different cut-off values can be used to more accurately determine the probability of DVT formation after hip and knee arthroplasty. Take preoperative measures to reduce the risk of DVT in the lower limbs after surgery. Prothrombin time, ESR, CRP, and age have great reference value in judging the formation of DVT in the lower limbs after hip replacement. It can evaluate the formation of DVT in the lower limbs after hip 
replacement and reduce the risk of thrombosis. Prothrombin time, ESR, CRP, and age still need to be further studied in the diagnosis of DVT after knee arthroplasty.

\section{Declarations}

\section{Acknowledgements}

Not applicable.

\section{Authors' contributions}

Study conception and design: Fan,Zhang,Sun. Acquisition of data: Fan,Duan,Wan,Yuan,Zhou. Analysis and interpretation of data: Fan,Qiu,Wenyi Li,Xicheng Li.Drafting of manuscript: Fan, Xicheng Li.Critical revision: Xicheng Li. All authors read and approved the final manuscript.

\section{Funding}

Not applicable.

\section{Availability of data and materials}

Not applicable.

\section{Ethics approval and consent to participate}

The study was approved by the Ethics Committee of Hebei Provincial People's Hospital,Shijiazhuang, China. All experiments do not involve human or animals.

\section{Consent for publication}

Not applicable.

\section{Competing interests}

This material has not been published and is not under consideration elsewhere. The authors declare that they have no competing interests. All authors have read and contributed to the submitted manuscript, and there is no conflict of interest among the authors.

\section{References}

1. Jiao Xiangxue, Cong Zihong, strong tension. Ultrasound-guided lower extremity nerve block for the analgesic, anti-inflammatory and antioxidant effects of elderly hip replacement surgery[J]. Journal of Hainan Medical University, 2018, 24(20): 1852-1855.

2. Chinese Medical Association Surgery Branch. Chinese general surgery perioperative thrombosis prevention and management guidelines[J]. Chinese Journal of Practical Surgery,2016,36(5):469-474. 
3. Wang Yuehua, Chen Feng, Zhou Kai, et al. Low-molecular-weight heparin calcium combined with physical methods to prevent perioperative lower limb deep vein thrombosis in elderly hip replacement [J]. Anhui Medicine, 2018, 22(12): 2414-2419.

4. Tang Xiao. Precautions for the application of plasma D-dimer in the diagnosis and treatment of venous thromboembolism [J]. Chinese Medical Journal, 2016, 51(4): 11-13.

5. Liu Yongyu, Xu Jingli, Lin Tianye, et al. Sensitivity and specificity of D-dimer in the diagnosis of chronic prosthetic infections after hip replacement [J]. Chinese Journal of Tissue Engineering Research, 2021, 12 (25): 1856.

6. Zhang Chengqing, Yang Xiaohong. Research progress in the diagnostic value of D-dimer in venous thromboembolism [J]. Xinjiang Medicine, 2019, 49(11): 1143-1146.

7. Jiang $Y$,Chu $X Y$,Chen $K$ P,et al.Clinical analysis of cerebral venous sinus thrombosis and its combined treatment of anticoagulation and endovascular thrombolysis[J]. Chin J Contemp Neurol Neurosurg, 2018, 17(12): 891-897.

8. Sugimoto E, Kuroda T, Fujita Y, et al. D-dimer testing cannot rule out thromboembolism after major lower extremity arthroplasties and thromboprophylaxis treatment[J]. J Anesth,2015,29(5):686689.DOI:10.1007/s00540-015-2020-1.

9. Durczynski A, Skulimowski A, Hogendorf P,et al.The concentration of D-dimers in portal blood positively correlates with overall survival in patients with non-resectable pancreatic cancer [J]. World J Surg Oncol, 2017,15(1):223-225.

10. Yang Jinghong. The diagnostic value of blood routine, erythrocyte sedimentation rate and C-reactive protein in children with acute pneumonia [J]. Chinese Medical Innovation, 2016, (5): 42-44. DOI: 10.3969/j.issn.1674-4985. 2016.05.011.

11. Peng HG区Xiagnostic value of c-reactive protein and myocardial enzyme in pediatric pneumonia[J].Maternal $\otimes$ Child Health Care of China,2015,30(21):3615.

12. Chen Xiaolan, Pan Lei, Wang Yong. The effectiveness of Padua Thrombosis Assessment Model for screening inpatients with deep vein thrombosis [J]. Chinese Journal of Internal Medicine, 2018, 57 (7): 515-516.

13. REED CR,FERGUSON RA,PENG Y,et al.Contact-isolation is a risk factor for venous thromboembolism in trauma patients[J].J Trauma Acute Care Surg,2015,79(5):833-83 7.

14. Chen Xiaolan, Pan Lei, Wang Yong. The effectiveness of Padua Thrombosis Assessment Model for screening inpatients with deep vein thrombosis [J]. Chinese Journal of Internal Medicine, 2018, 57 (7): 516.

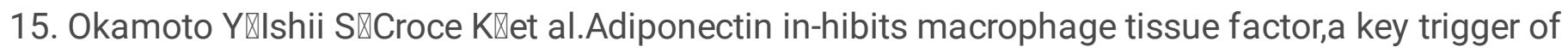
thrombosis in disrupted atherosclerotic plaques[J].Atherosclerosis,2013,226(2):373.

16. WAKABAYASHI H,HASEGAWA M,NIIMI R,et al.Clinical analysis of preoperative deep vein thrombosis risk factors in patients undergoing total hip arthroplasty[J]. Thromb Res,2015,136(5):855-858.

17. AhI V『Hu HMखHenke PKखet al $\triangle A$ validation study of aretrospective venous throlllboembolism risk scoring method[J].Ann surg $₫ 2010 \otimes 251$ (2)『344-350.DOI: 10.1111/j.1432-2277.2010.01105. 


\section{Tables}

Table 1

Comparison of incidence of lower limb venous thrombosis after different replacement types

\begin{tabular}{|c|c|c|c|c|}
\hline $\begin{array}{l}\text { displacement } \\
\text { type }\end{array}$ & $\begin{array}{l}\text { number of } \\
\text { cases }\end{array}$ & $\begin{array}{l}\text { Number of cases of deep vein thrombosis in } \\
\text { lower extremity (\%) }\end{array}$ & $\mathrm{X} 2$ & $P$ \\
\hline THA & 35 & $8(22.9 \%)$ & \multirow[t]{4}{*}{13.294} & \multirow[t]{4}{*}{0.004} \\
\hline $\mathrm{HA}$ & 73 & $34(46.6 \%)$ & & \\
\hline TKA & 77 & $25(32.5 \%)$ & & \\
\hline UKA & 31 & $19(61.3 \%)$ & & \\
\hline
\end{tabular}

Table 2

General information of 172 patients

\begin{tabular}{|c|c|c|c|c|c|c|c|c|}
\hline \multirow[t]{2}{*}{ group } & \multirow{2}{*}{$\begin{array}{l}\text { number of } \\
\text { cases }\end{array}$} & \multicolumn{2}{|c|}{ gender } & \multirow[t]{2}{*}{ Age (years) } & \multicolumn{4}{|c|}{ displacement type } \\
\hline & & male & female & & TKA & UKA & THA & HA \\
\hline Thrombus group & 86 & 23 & 63 & $\begin{array}{l}70.98 \pm \\
10.22\end{array}$ & 25 & 19 & 8 & 34 \\
\hline $\begin{array}{l}\text { The control } \\
\text { group }\end{array}$ & 86 & 23 & 63 & $64.00 \pm 6.39$ & 47 & 11 & 21 & 7 \\
\hline
\end{tabular}

Table 3

Comparison of D-dimer, albumin and hemoglobin levels between thrombus group and control group

\begin{tabular}{|llll|}
\hline group & D-dimer $(\mathrm{mg} / \mathbf{L})$ & Albumin $(\mathbf{g} / \mathbf{L})$ & Hemoglobin $(\mathbf{g} / \mathbf{L})$ \\
\hline Thrombus group & $0.98(0.378 \sim 3.463)$ & $38.806 \pm 4.625$ & $128.663 \pm 15.776$ \\
\hline The control group & $0.43(0.238 \sim 0.893)$ & $41.003 \pm 3.824$ & $134.430 \pm 12.842$ \\
\hline statistics & $\mathrm{Z}=-4.196$ & $\mathrm{t}=3.395$ & $\mathrm{t}=2.629$ \\
\hline $\mathrm{P}$ & 0.000 & 0.001 & 0.009 \\
\hline
\end{tabular}


Table 4

Comparison of D-mer, albumin, and hemoglobin levels of hip and knee replacements in thrombosis group

\begin{tabular}{|llll|}
\hline group & D-dimer $(\mathrm{mg} / \mathrm{L})$ & Albumin $(\mathrm{g} / \mathrm{L})$ & Hemoglobin $(\mathrm{g} / \mathrm{L})$ \\
\hline Hip arthroplasty & $3.25(1.240 \sim 8.350)$ & $36.199 \pm 4.569$ & $125.897 \pm 16.665$ \\
\hline Knee arthroplasty & $0.45(0.290 \sim 0.970)$ & $40.969 \pm 3.4269$ & $130.957 \pm 14.785$ \\
\hline statistics & $\mathrm{Z}=-5.830$ & $\mathrm{t}=5.527$ & $\mathrm{t}=1.491$ \\
\hline $\mathrm{P}$ & 0.000 & 0.000 & 0.140 \\
\hline
\end{tabular}

Figures

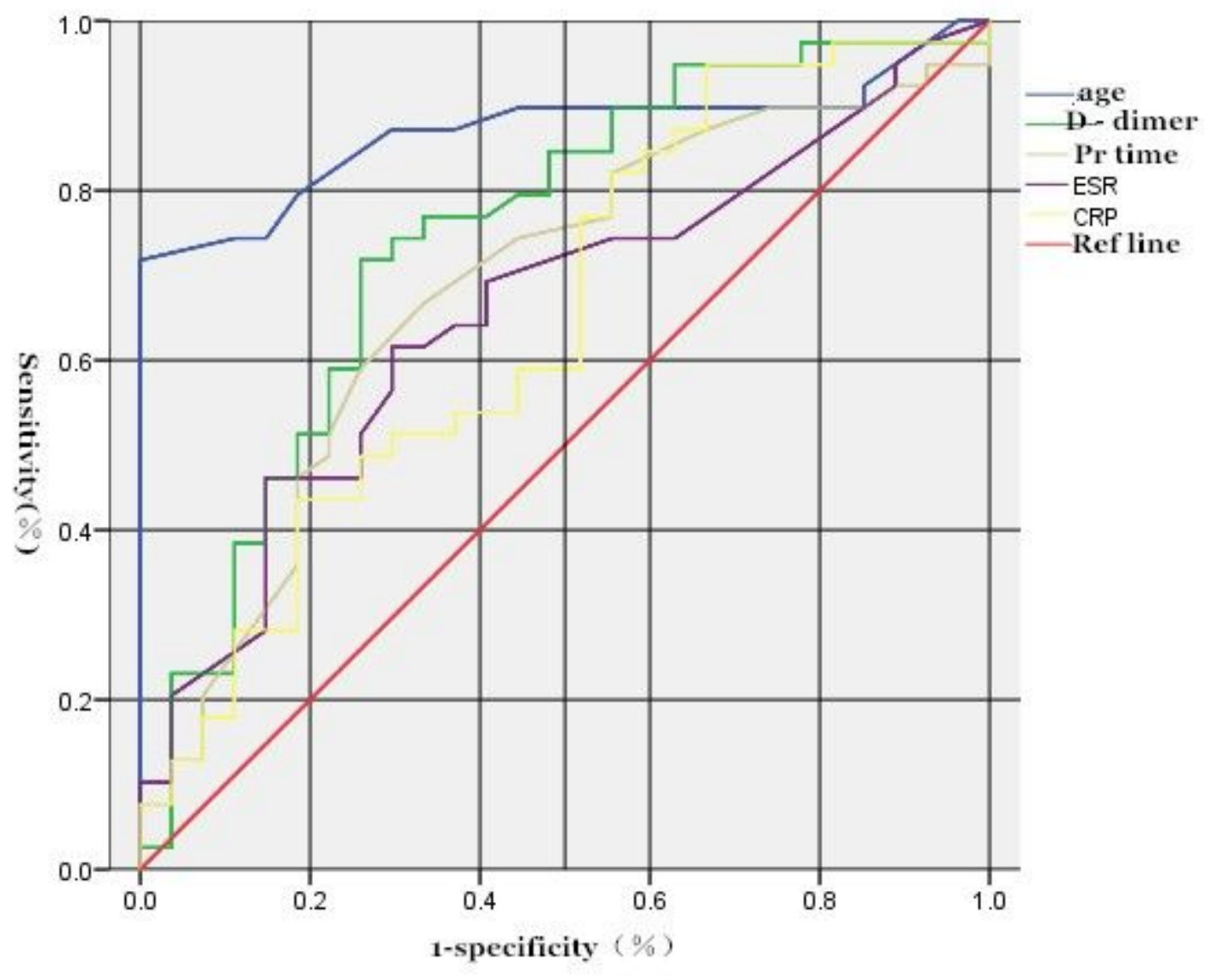

Figure 1

Subject ROC curve of D-dimer, prothrombin time, ESR, CRP, age diagnosis of lower limb DVT after hip replacement 


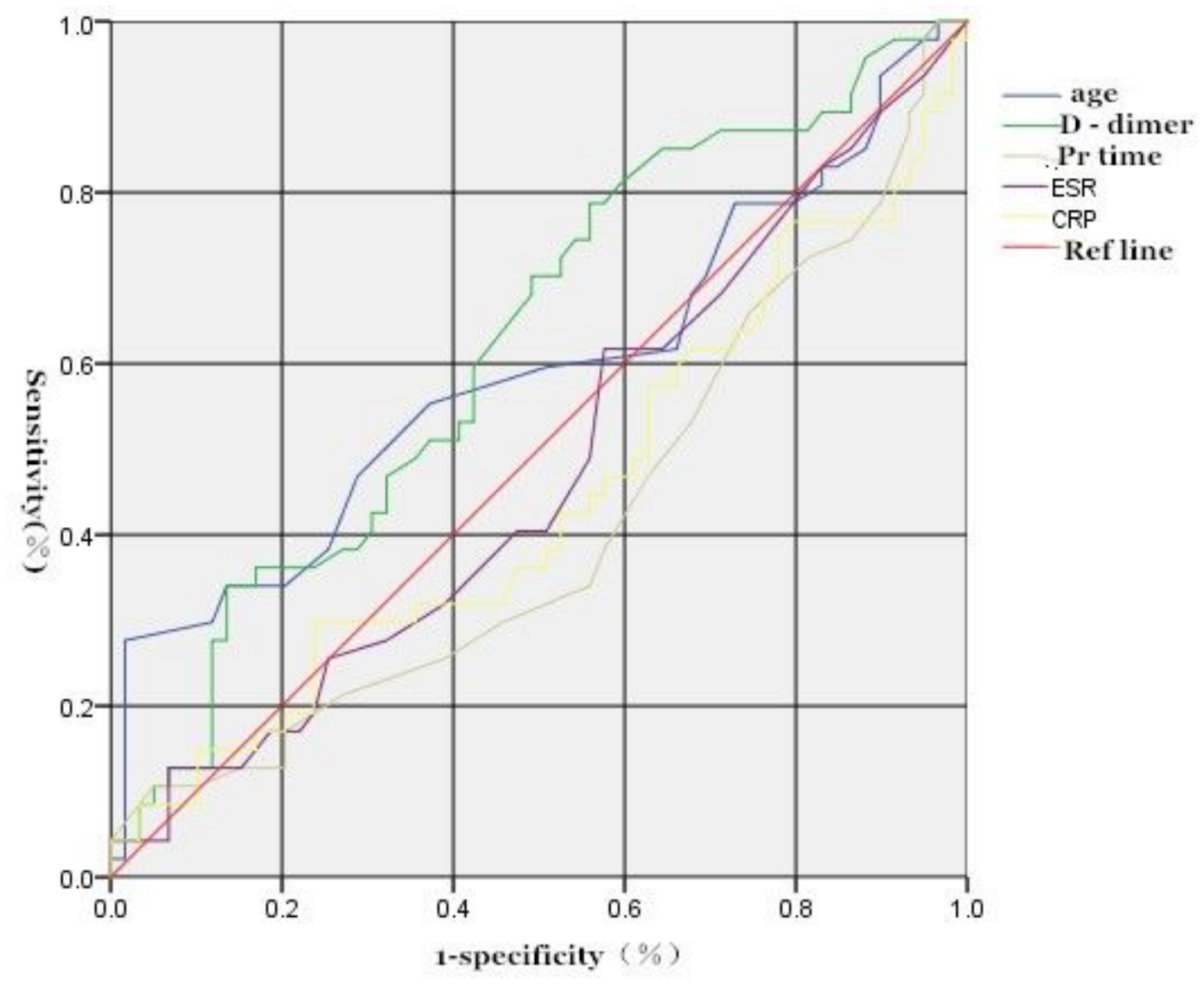

Figure 2

Subject ROC curve of D-dimer, prothrombin time, ESR, CRP, age diagnosis of lower limb DVT after knee arthroplasty 